\title{
EC Transactions Use Different Web-Based Platforms
}

\author{
Whe Dar Lin \\ The Overseas Chinese Institute of Technology \\ Dept of Information Management, \\ No. 100, Chiao Kwang Road Taichung 40721, Taiwan
}

\begin{abstract}
A review of the methodological aspects of the EC transactions use different Web-based platforms is presented. Our design techniques for complex EC environments are present to avoid the viruses infected EC transaction files. When an executive service code is ordered it may get infected with some viruses before the signature is attached to it. The infected EC transaction files cannot be detected by signature verification and the origin of the infection order cannot be specified by different Web-based platforms. Applied our EC transactions use different Web-based platforms, these techniques lead to an EC framework with clients, agents, EC application servers and EC system management. The paper discusses how Web-based platforms can use signature method together with detection infected in the preprocessing step enable us to specify the origin of the infection side. The impact of our proposed method can improve Web-based platforms relationships between EC transaction agents.
\end{abstract}

\section{Introduction}

In EC era, there has been a general trend towards partnership Web-based platforms together with a reduction and consolidation of the supply based in order to have better EC application relationships with fewer Web-based platforms. It is an important step that an execution service code can be detected by checking the consistency of original transaction order with its accompanying digital signature [1], [2], [5], [6], [8]. The cooperative Web-based platforms approach to supplier is characterized by long term contracts, integrated key protocols, share marketing database, and a commitment to partner relationships.

We don't think this is a good architecture using in Web-based platform. [12][13][14] Some other efforts through the directory services, name services, library service to reduce complex about viruses impaction. This approach yields an efficient solution for simple and static point-to-point interaction in collaborative EC transaction order. Nevertheless, we need face dynamic communication commit from different Web-based platforms for multiple relationships between one another on supply chain management. We will choice the ID-based scheme [10], a large publickey file is not required because each user's public key is nothing but an identity and communication cost is low using in EC applications for different Web-based platforms.

Our proposed signature method is based on a public key scheme. We assume that special entities, such as transaction suppliers in supply chain are not reliable for 
concerning the signature forgery or computer viruses. The rest of this paper is organized as follows. Sect. 2 gives some general assumptions about viruses and transaction supplier and Web-based platform. The proposed our security Web-based platform scheme will be described in Sect. 3 and Sect. 4 the correctness shall be discussion. Our proposed scheme security analysis is described in Sect. 5, and we discuss some implementation Web-based platform issue in Sect. 6. We conclude with some final remarks shall be stated in Sect.7.

\section{Assumptions and Model}

Here, we provide several assumptions about viruses and transaction supplier, and security in general using in our protocol. A protocol is a set of rules and conventions that define the communication framework between two or more EC transactions. After that, we shall describe the Web-based platforms.

\subsection{General Assumptions about Viruses and Transaction Supplier}

Our proposed scheme relies on the existence of a hash function h. Specifically,

Assumption 1: We assume there exist a function h such that:

On random input $\left(\mathrm{r}_{\mathrm{i}}, \mathrm{m}_{\mathrm{i}}\right)$, it is difficult to generate $\left(\mathrm{e}_{\mathrm{i}}\right)$ such that $\mathrm{h}\left(\mathrm{r}_{\mathrm{i}}, \mathrm{m}_{\mathrm{i}}\right)=\left(\mathrm{e}_{\mathrm{i}}\right)$. More generally, it is difficult to generate such $\left(e_{i}\right)$ on input $\left(r_{i}, m_{i}\right)$ and samples of signature on random messages signed with EC-based platforms.

Assumption 2: Web-based platform act according to the following conditions:

- They create transaction supplier honestly in marketing channels.

- They do not refuse to reply to the requests or questions from the EC agent.

- Viruses can infect and incubate warehouse files and EC order files on Web-based platforms.

- Viruses can damage both warehouse files and EC order files on Web-based platforms.

Our proposed automatic signature scheme of protection against viruses works upon the following security assumptions [1], [2], [3], [4], [9], [11]:

- Solving the discrete logarithm problem is difficult, so we use in cryptographic protocols.

- Inverse calculation of one-way hash functions is difficult that we use assumption is often made in cryptographic protocols.

- Distributed operating system in Web-based executes a verification program properly. This assumption is important because improper events must be ruled out in the verification procedure so that one can rely on the results of the verification. 


\subsection{Client-Server Transaction Model for Procurement}

In this paper, the transaction supplier on the Web-based platform will reduce the amount of complete purchased work items and the turnaround time the deliver the order transaction services.

- Creates the service system library, good library, customer library and included files,

- Signs all library files following every subroutine, procedure and initial data structure,

- Calculates the fingerprints of the included files with a one-way hash function and creates the database of the fingerprints (fingerprint data file),

- Computes proxy integers and attaches them to the Web-based platform,

- Creates the signatures for the shops on Web-based platform and the fingerprint data file.

The included files and customer relation data are text files and are accessed during preprocessing. The transaction includes all processes from preprocessing to linking. The fingerprint of an included file is an output of a one-way hash function taking the included file as an input file. The fingerprint data file is composed of pairs of the names of the included files and their fingerprints. From this file, one can authenticate the validity of the included files.

\section{Our Proposed Automatic Signature Scheme}

In this section, we shall illustrate the structure of our proposed method. In our proposed protocol, The transaction supplier is denoted by $u_{m}$, the EC server $u_{s}$ and the mobile user, client $\mathrm{u}_{\mathrm{c}}$.

They follow the steps below.

Step 1: The EC server $\mathrm{u}_{\mathrm{s}}$ sends the request the Web-based platform $\mathrm{R}$ to the transaction supplier $\mathrm{u}_{\mathrm{m}}$.

Step 2: The transaction supplier $\mathrm{u}_{\mathrm{m}}$ sends the Web-based platform $\mathrm{R}$ to the EC server $\mathrm{u}_{\mathrm{s}}$

Step 3: The mobile user, client $\mathrm{u}_{\mathrm{c}}$ sends the request the Web-based platform $\mathrm{R}$ for the transaction order $\mathrm{P}$ to the EC server $\mathrm{u}_{\mathrm{s}}$.

Step 4:The EC server $u_{s}$ sends the executable transaction bill service code $M$ to the client $u_{c}$.

\subsection{The Initialization Phase}

The system parameter are listed as follows:

(1)We use a prime $\mathrm{p}$ with $2^{511}<\mathrm{p}<2^{512}$, a prime divisor $\mathrm{qlp}-1$, a generator $\mathrm{g}$ with order q over $\mathrm{GF}(\mathrm{p})$, and

(2) the security information of the transaction supplier $\mathrm{u}_{\mathrm{m}}: \mathrm{x}_{\mathrm{m}}$

(3) the public information of the transaction supplier $\mathrm{u}_{\mathrm{m}}: \mathrm{y}_{\mathrm{m}}$ 


$$
y_{m} \equiv g^{x_{m}} \bmod p
$$

(4) the security information of the EC server $u_{s}: x_{s}$

(5) the public information of the EC server $u_{s}: y_{s}$,

$$
y_{s} \equiv g^{x_{s}} \bmod p
$$

(6) the security information of the mobile user, client $u_{c}: x_{c}$

(7) the public information of the mobile user, client $u_{c}: y_{c}$,

$$
y_{c} \equiv g^{x_{c}} \bmod p
$$

\subsection{The EC Server Requests the Delivery of a Web-Based Platform}

When a server $\mathrm{u}_{\mathrm{s}}$ requests the delivery of a Web-based platform named $\mathrm{R}$. The server sends his secret key $\mathrm{x}_{\mathrm{s}}$ along with four integers calculated from a random number $\mathrm{k}_{1}$. The computation is as follows.

(1). Generate a random number $\mathrm{k}_{1}$ satisfying $0<\mathrm{k}_{1}<\mathrm{q}-1$

(2). Compute

$$
\begin{gathered}
\mathrm{r}_{1} \equiv \mathrm{g}^{\mathrm{k} 1} \bmod \mathrm{p} \\
\mathrm{m}_{1} \equiv \mathrm{u}_{\mathrm{s}} \| \mathrm{R} \bmod \mathrm{p} \\
\mathrm{e}_{1} \equiv \mathrm{h}\left(\mathrm{r}_{1}, \mathrm{~m}_{1}\right) \bmod \mathrm{q} \\
\mathrm{s}_{1} \equiv \mathrm{k}_{1}-\mathrm{x}_{\mathrm{s}} \mathrm{e}_{1} \bmod \mathrm{q}
\end{gathered}
$$

where $\mathrm{h}$ is a one-way function and $\|$ denotes concatenation.

(3). Send $\left(\mathrm{u}_{\mathrm{s}}, \mathrm{R}, \mathrm{e}_{1}, \mathrm{~s}_{1}\right)$ with the request to the transaction supplier $\mathrm{u}_{\mathrm{m}}$.

\subsection{The Transaction Supplier Sends the Web-Based Platform to the EC Server}

When the transaction supplier $\mathrm{u}_{\mathrm{m}}$ receives the integers $\left(\mathrm{u}_{\mathrm{s}}, \mathrm{R}, \mathrm{e}_{1}, \mathrm{~s}_{1}\right)$, he verifies the server $u_{s}$ requests and then delivers a Web-based platform $C_{R}$. The transaction supplier $\mathrm{u}_{\mathrm{m}}$ verifies $\left(\mathrm{u}_{\mathrm{s}}, \mathrm{R}, \mathrm{e}_{1}, \mathrm{~s}_{1}\right)$ by computing the following equations.

(1). compute

$$
\mathrm{r}^{*} \equiv \mathrm{g}^{\mathrm{sl}} \mathrm{y}_{\mathrm{s}}{ }^{\mathrm{el}}
$$

check whether the congruence $e_{1}=h\left(r^{*}, u_{s} \| R\right)$ holds.

If it holds, the transaction supplier $\mathrm{u}_{\mathrm{m}}$ does as follow.

(2). Generate a random number $\mathrm{k}_{2}$ satisfying $0<\mathrm{k}_{2}<\mathrm{q}-1$

(3). Compute

$$
\mathrm{r}_{2} \equiv \mathrm{g}^{\mathrm{k} 2} \bmod \mathrm{p}
$$




$$
\begin{gathered}
\mathrm{t}_{\mathrm{m}} \equiv \mathrm{x}_{\mathrm{m}}+\mathrm{k}_{2} * \mathrm{r}_{2} \bmod \mathrm{q} \\
\mathrm{m}_{2} \equiv \mathrm{C}_{\mathrm{R}} \| \mathrm{t}_{\mathrm{m}} \bmod \mathrm{p} \\
\mathrm{e}_{2} \equiv \mathrm{h}\left(\mathrm{r}_{2}, \mathrm{~m}_{2}\right) \bmod \mathrm{q} \\
\mathrm{s}_{2} \equiv \mathrm{k}_{2}-\mathrm{x}_{\mathrm{m}} \mathrm{e}_{2} \bmod \mathrm{q}
\end{gathered}
$$

(4). Send the Web-based platform $C_{R}$ and its signature $\left(C_{R}, t_{m}, e_{2}, s_{2}\right)$ to $u_{s}$

\subsection{The Client Requests to the EC Server to Send Service Code for an Order P}

A client user $u_{c}$ wants to get a service code for an order $P$, and the server creates an executable service code $\mathrm{M}$ and its signature. When the server $\mathrm{u}_{\mathrm{s}}$ receives the integers $\left(C_{R}, t_{m}, e_{2}, s_{2}\right)$, he verifies that a server $u_{m}$ sends then accept the Web-based platform $C_{R}$. The server $u_{s}$ verifies $\left(C_{R}, t_{m}, e_{2}, s_{2}\right)$ by computing the following equations.

(1). Compute

$$
\mathrm{r}^{*} \equiv \mathrm{g}^{\mathrm{s} 2} \mathrm{y}_{\mathrm{m}}^{\mathrm{e} 2}
$$

check whether the congruence $\mathrm{e}_{2}=\mathrm{h}\left(\mathrm{r}^{*}, \mathrm{C}_{\mathrm{R}} \| \mathrm{t}_{\mathrm{m}}\right)$ holds. If it holds, the server $\mathrm{u}_{\mathrm{s}}$ does as the client wishes. In the process of calculating a signature, the client executes the following steps:

(2). Generate a random number $\mathrm{k}_{3}$ satisfying $0<\mathrm{k}_{3}<\mathrm{q}-1$

(3). Compute

$$
\begin{gathered}
\mathrm{r}_{3} \equiv \mathrm{g}^{\mathrm{k} 3} \bmod \mathrm{p} \\
\mathrm{m}_{3} \equiv \mathrm{u}_{\mathrm{c}} \| \mathrm{P} \bmod \mathrm{p} \\
\mathrm{e}_{3} \equiv \mathrm{h}\left(\mathrm{r}_{3}, \mathrm{~m}_{3}\right) \bmod \mathrm{q} \\
\mathrm{s}_{3} \equiv \mathrm{k}_{3}-\mathrm{x}_{\mathrm{s}} \mathrm{e}_{3} \bmod \mathrm{q}
\end{gathered}
$$

(4). Send $\left(u_{c}, P, e_{3}, s_{3}\right)$ with the request to the server $u_{s}$.

\subsection{The Server Processes the Order and Sends the Execution Service Code to the Client}

When the server $u_{s}$ receives the integers $\left(u_{c}, P, e_{3}, s_{3}\right)$, he verifies that a client $u_{c}$ requests that the Web-based platform $C_{R}$ processes the order $P$. The server $u_{s}$ verifies $\left(\mathrm{u}_{\mathrm{c}}, \mathrm{P}, \mathrm{e}_{3}, \mathrm{~s}_{3}\right)$ by computing the following equations (1). compute

$$
r^{*} \equiv g^{s 3} y_{s}^{e 3} \bmod p
$$


check whether the congruence $\mathrm{e}_{3}=\mathrm{h}\left(\mathrm{r}^{*}, \mathrm{u}_{\mathrm{c}} \| \mathrm{P}\right)$ holds. If it holds, in the process of calculating a signature, the server processes the order $\mathrm{P}$ and executes the following steps:

(2). Generate a random number $\mathrm{k}_{4}$, satisfying $0<\mathrm{k}_{4}<\mathrm{q}-1$

(3). Compute

$$
\begin{gathered}
\mathrm{r}_{4} \equiv \mathrm{Pg}^{-\mathrm{k} 4} \bmod \mathrm{p} \\
\mathrm{s}_{4} \equiv \mathrm{k}_{4}-\mathrm{r}_{4} \mathrm{x}_{\mathrm{s}} \bmod \mathrm{q} \\
\mathrm{M} \equiv \mathrm{C}_{\mathrm{R}}(\mathrm{P}) \bmod \mathrm{p} \\
\mathrm{m}_{4} \equiv \mathrm{u}_{\mathrm{c}}\|\mathrm{P}\| \mathrm{M} \bmod \mathrm{p} \\
\mathrm{e}_{4} \equiv \mathrm{h}\left(\mathrm{r}_{3}, \mathrm{~m}_{3}\right) \bmod \mathrm{q} \\
\mathrm{t}_{\mathrm{s}} * \mathrm{~h}(\mathrm{M}) \equiv \mathrm{t}_{\mathrm{m}}+\mathrm{r}_{2} \mathrm{x}_{\mathrm{s}} \bmod \mathrm{q} \\
\mathrm{r}_{2} \equiv \mathrm{g}^{\mathrm{s} 2} \mathrm{y}_{\mathrm{m}}{ }^{\mathrm{e} 2} \bmod \mathrm{p}
\end{gathered}
$$

(4). Send $\left(r_{4}, s_{4}, t_{s}, M\right)$ with the request to the server $u_{s}$.

The Web-based platform can be used only by the server $u_{s}$ because the integer $t_{m}$ is the proxy key of the transaction supplier $u_{m}$. However, if another user happens to know $u_{\mathrm{m}}$ 's secret key, then he can use this $\mathrm{u}_{\mathrm{m}}$ 's Web-based platform and create a bad executable service code with this proper proxy. Then, the executable service code may damage someone's computer. The basic rule for this event is that the owner $u_{m}$ of the Web-based platform storing the proxy integer key $t_{m}$ is responsible for the damage so that the transaction supplier must hide his secret key.

\subsection{Signature Verification}

When a user executes a service code $M$ made by $u_{s}$, the use verifies the signature with the transaction supplier's public key and server's public key. That he checks whether the signature satisfies the congruence such that

$$
g^{t_{s} h(M)} \equiv y_{m}\left(r_{2} y_{s}\right)^{r_{2}} \bmod p
$$

Since the signature $\left(\mathrm{r}_{4}, \mathrm{~s}_{4}, \mathrm{t}_{\mathrm{s}}, \mathrm{M}\right)$ is automatically created, an infection of a generated executable service code can be detected in the above verification process. Using a signature can possibly be created for a contaminated executable service code, and in this case, the infection can be detected by the verification. We can determine who causes the injection. 


\section{Correctness of Our Scheme}

\section{Theorem 1:}

1. The transaction supplier $\mathrm{u}_{\mathrm{m}}$ can get the correctness request from the server $\mathrm{u}_{\mathrm{s}}$

2. The server $u_{s}$ can get the correctness from transaction supplier $u_{m}$.

3 . The server $u_{s}$ can get the correctness request from client $u_{c}$.

Proof:

(1) The transaction supplier $u_{m}$, receiving $\left(u_{s}, R, e_{1}, s_{1}\right)$ from $u_{s}$ can compute

$r^{*}=g^{s 1} y_{s}^{e 1}=g^{k 1-x s e 1} y_{s}^{e 1}=g^{k 1-x s e 1} g^{\text {xsel }}=g^{k 1}=r_{1}$

$\mathrm{h}\left(\mathrm{r}^{*}, \mathrm{u}_{\mathrm{s}} \| \mathrm{R}\right)=\mathrm{h}\left(\mathrm{r}_{1}, \mathrm{u}_{\mathrm{s}} \| \mathrm{R}\right)=\mathrm{h}\left(\mathrm{r}_{1}, \mathrm{~m}_{1}\right)=\mathrm{e}_{1}$

Then we can be sure the signature of $\left(u_{s}, R\right)$ is $\left(e_{1}, s_{1}\right)$

(2) The server $u_{s}$, receiving $\left(C_{r}, t_{m}, e_{2}, s_{2}\right)$ from $u_{m}$ can compute

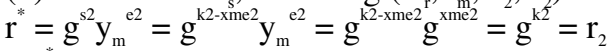

$\mathrm{h}\left(\mathrm{r}^{*}, \mathrm{C}_{\mathrm{r}} \| \mathrm{t}_{\mathrm{m}}\right)=\mathrm{h}\left(\mathrm{r}_{2}, \mathrm{C}_{\mathrm{r}} \| \mathrm{t}_{\mathrm{m}}\right)=\mathrm{h}\left(\mathrm{r}_{2}, \mathrm{~m}_{2}\right)=\mathrm{e}_{2}$

Then we can be sure the signature of $\left(\mathrm{C}_{\mathrm{r}} \| \mathrm{t}_{\mathrm{m}}\right)$ is $\left(\mathrm{e}_{2}, \mathrm{~s}_{2}\right)$

(3) The server $u_{s}$, receiving $\left(u_{c}, P, e_{3}, s_{3}\right)$ from client $u_{c}$ can compute

$\mathrm{r}^{*}=\mathrm{g}^{\mathrm{s} 3} \mathrm{y}_{\mathrm{c}}^{\mathrm{e} 3}=\mathrm{g}^{\mathrm{k} 3-\mathrm{xcc3}} \mathrm{y}_{\mathrm{c}}^{\mathrm{e} 3}=\mathrm{g}^{\mathrm{k} 3-\mathrm{xce} 3} \mathrm{~g}^{\mathrm{xce3}}=\mathrm{g}^{\mathrm{k} 3}=\mathrm{r}_{3}$

$\mathrm{h}\left(\mathrm{r}^{*}, \mathrm{u}_{\mathrm{c}} \| \mathrm{P}\right)=\mathrm{h}\left(\mathrm{r}_{3}, \mathrm{u}_{\mathrm{c}} \| \mathrm{P}\right)=\mathrm{h}\left(\mathrm{r}_{3}, \mathrm{~m}_{3}\right)=\mathrm{e}_{3}$

Then we can be sure the signature of $\left(u_{c}, P\right)$ is $\left(e_{3}, s_{3}\right)$

Theorem 2:The server $u_{s}$ can processes the correctness transaction order request from the client $u_{\mathrm{c}}$.

Proof: The client $\mathrm{u}_{\mathrm{c}}$, receiving $\left(\mathrm{r}_{4}, \mathrm{~s}_{4}, \mathrm{t}_{\mathrm{s}}, \mathrm{M}\right)$ from server $\mathrm{u}_{\mathrm{s}}$ can compute $g^{t_{s} h(M)} \equiv y_{m}\left(r_{2} y_{s}\right)^{r_{2}}$

where $g^{t_{s} h(M)} \equiv g^{t_{m}+x_{s} r_{2}} \equiv g^{x_{m}+k_{2} r_{2}+x_{s} r_{2}} \equiv y_{m} r_{2}^{r_{2}} y_{s}^{r_{2}} \equiv y_{m}\left(r_{2} y_{s}\right)^{r_{2}}$ Then we can be sure the signature of $\mathrm{M}$ is $\left(\mathrm{t}_{\mathrm{s}}, \mathrm{M}\right)$ and use the public key of transaction supplier and server.

\section{Security Considerations}

Theorem 3: The crackers want to reveal the secret key from public key is computing impossible.

Proof: The crackers want to from the public key $\mathrm{y}_{\mathrm{m}} \equiv \mathrm{g}^{\mathrm{xm}} \bmod \mathrm{p}, \mathrm{y}_{\mathrm{s}} \equiv \mathrm{g}^{\mathrm{xs}} \bmod \mathrm{p}$, and $\mathrm{y}_{\mathrm{c}}$ $\equiv \mathrm{g}^{\mathrm{xc}} \bmod \mathrm{p}$ to reveal $\mathrm{x}_{\mathrm{m}}, \mathrm{x}_{\mathrm{s}}$, and $\mathrm{x}_{\mathrm{c}}$, they have to solve the discrete logarithm problem.

Theorem 4: The malicious users want to forger the request from the proposed scheme is computing impossible.

(1) The transaction supplier $u_{m}$ get the false receive from malicious users.

(2) The server $u_{s}$ can get the false request from malicious users. 
Proof:

(1) The transaction supplier can use $\left(\mathrm{u}_{\mathrm{s}}, \mathrm{R}, \mathrm{e}_{1}, \mathrm{~s}_{1}\right)$ to verify

$\mathrm{r}^{*}=\mathrm{g}^{\mathrm{s} 1} \mathrm{y}_{\mathrm{s}}^{\mathrm{el}}=\mathrm{g}^{\mathrm{k} 1-\mathrm{xse} 1} \mathrm{y}_{\mathrm{s}}^{\mathrm{el}}=\mathrm{g}^{\mathrm{k1}-\mathrm{xsel} 1} \mathrm{~g}^{\mathrm{xsel}}=\mathrm{g}^{\mathrm{k} 1}=\mathrm{r}_{1}$, But the malicious users construct $\left(\mathrm{u}_{\mathrm{s}}, \mathrm{R}, \mathrm{e}_{1}{ }^{\mathrm{r}}\right.$, $\mathrm{s}_{1}{ }^{\prime}$ ) without know $\mathrm{x}_{\mathrm{s}}$, the congruence, $\mathrm{r}^{*}=\mathrm{g}^{\mathrm{s}{ }^{\prime}} \mathrm{y}_{\mathrm{s}}^{\mathrm{el}}=\mathrm{r}_{1}$. They have to solve the discrete logarithm problem.

(2) The proof method is similar as part (1).

Theorem 5: The malicious users want to forger the signature from the proposed scheme is computing impossible.

(1) The server $u_{s}$ can get the false Web-based platform from malicious user.

(2) The client $u_{c}$ can get the false executable service code from malicious user.

(1) Proof: The server $u_{s}$ the receive $\left(C_{r}, t_{m}, e_{2}, s_{2}\right)$ from $u_{m}$ can compute

$\mathrm{r}^{*}=\mathrm{g}^{\mathrm{s} 2} \mathrm{y}_{\mathrm{m}}{ }^{\mathrm{2} 2}=\mathrm{g}^{\mathrm{k} 2 \text {-xme2 }} \mathrm{y}_{\mathrm{m}} \mathrm{e}^{2}=\mathrm{g}^{\mathrm{k} 2 \mathrm{-xme} 2} \mathrm{~g}^{\mathrm{xme2}}=\mathrm{g}^{\mathrm{k} 2}=\mathrm{r}_{2}$

$\mathrm{h}\left(\mathrm{r}^{*}, \mathrm{C}_{\mathrm{r}} \| \mathrm{t}_{\mathrm{m}}\right)=\mathrm{h}\left(\mathrm{r}_{2}, \mathrm{C}_{\mathrm{r}} \| \mathrm{t}_{\mathrm{m}}\right)=\mathrm{h}\left(\mathrm{r}_{2}, \mathrm{~m}_{2}\right)=\mathrm{e}_{2}$

We will sure the signature of $\left(\mathrm{C}_{\mathrm{r}} \|_{\mathrm{m}}\right)$ is $\left(\mathrm{e}_{2}, \mathrm{~s}_{2}\right)$

Case I: The malicious users use the false $\left(\mathrm{C}_{\mathrm{r}}, \mathrm{t}_{\mathrm{m}}, \mathrm{e}_{2}, \mathrm{~s}_{2}\right)$ signature send to server $\mathrm{u}_{\mathrm{s}}$

$\mathrm{r}^{*}=\mathrm{g}^{\mathrm{s} 2} \mathrm{y}_{\mathrm{m}}^{\mathrm{e} 2}=\mathrm{g}^{\mathrm{k} 2 \mathrm{-xme} 2} \mathrm{y}_{\mathrm{m}}{ }^{\mathrm{e} 2}=\mathrm{g}^{\mathrm{k} 2-\mathrm{xme} 2} \mathrm{~g}^{\mathrm{xme} 2}=\mathrm{g}^{\mathrm{k} 2}=\mathrm{r}_{2}$

$\mathrm{h}\left(\mathrm{r}^{*}, \mathrm{C}_{\mathrm{r}}^{\prime} \| \mathrm{t}_{\mathrm{m}}\right)=\mathrm{h}\left(\mathrm{r}_{2}, \mathrm{C}_{\mathrm{r}}^{\prime} \| \mathrm{t}_{\mathrm{m}}\right)=\mathrm{h}\left(\mathrm{r}_{2}, \mathrm{~m}^{\prime}\right)=\mathrm{e}^{\prime} \neq \mathrm{e}_{2}$

We will sure the signature of $\left(\mathrm{C}_{\mathrm{r}}, \mathrm{t}_{\mathrm{m}}, \mathrm{e}_{2}, \mathrm{~s}_{2}\right)$ is false signature.

Case II: The malicious users use the false $\left(\mathrm{C}_{\mathrm{r}}, \mathrm{t}_{\mathrm{m}}, \mathrm{e}_{2}, \mathrm{~s}_{2}\right)$ signature send to server $\mathrm{u}_{\mathrm{s}}$

$\mathrm{r}^{*}=\mathrm{g}^{\mathrm{s} 2} \mathrm{y}_{\mathrm{m}}^{\mathrm{e} 2}=\mathrm{g}^{\mathrm{k} 2 \mathrm{-xme} 2} \mathrm{y}_{\mathrm{m}}{ }^{\mathrm{e} 2}=\mathrm{g}^{\mathrm{k} 2-\mathrm{xme} 2} \mathrm{~g}^{\mathrm{xme} 2}=\mathrm{g}^{\mathrm{k} 2}=\mathrm{r}_{2}$

$\mathrm{h}\left(\mathrm{r}^{*}, \mathrm{C}_{\mathrm{r}} \| \mathrm{t}_{\mathrm{m}}{ }^{\prime}\right)=\mathrm{h}\left(\mathrm{r}_{2}, \mathrm{C}_{\mathrm{r}} \| \mathrm{t}_{\mathrm{m}}{ }^{\prime}\right)=\mathrm{h}\left(\mathrm{r}_{2}, \mathrm{~m}^{\prime}\right)=\mathrm{e}^{\prime} \neq \mathrm{e}_{2}$

We will sure the signature of $\left(\mathrm{C}_{\mathrm{r}}, \mathrm{t}_{\mathrm{m}}, \mathrm{e}_{2}, \mathrm{~s}_{2}\right)$ is false signature.

Case III: The malicious users use the false $\left(\mathrm{C}_{\mathrm{r}}{ }^{\prime}, \mathrm{t}_{\mathrm{m}}{ }^{\prime}, \mathrm{e}_{2}{ }^{\prime}, \mathrm{s}_{2}{ }^{\prime}\right)$ signature send to server $\mathrm{u}_{\mathrm{s}}$ $\mathrm{r}^{*}=\mathrm{g}^{\mathrm{s}{ }^{2}} \mathrm{y}_{\mathrm{m}}{ }^{\mathrm{e}}{ }^{\prime}=\mathrm{r}_{2}{ }^{\prime}, \mathrm{h}\left(\mathrm{r}_{2}{ }^{\prime}, \mathrm{C}_{\mathrm{r}}{ }^{\prime} \mathrm{It}_{\mathrm{m}}{ }^{\prime}\right)=\mathrm{e}_{2}{ }^{\prime}$

But the malicious users choose another Web-based platform $\mathrm{C}_{\mathrm{r}}$, and another proxy key $\mathrm{t}_{\mathrm{m}}$, without know $\mathrm{x}_{\mathrm{m}}$, the congruence equation that they have to solve the discrete logarithm problem.

(2) The proof method is similar as part (1).

Theorem 6: The computational complexity for an intruder to cryptanalyze a new session key in our scheme, after having received previous transaction order data, the public key, the old messages and the new message is as hard to cryptanalyze a plaintext in the ElGamal scheme when the order of $\mathrm{g}$ is a prime.

Proof. Let $\mathrm{B}_{\mathrm{O}}$ correspond with the problem of breaking our scheme and $\mathrm{B}_{\mathrm{E}}$ with breaking the ElGamal encryption scheme with the order of $\mathrm{g}$ is prime. There is an existence of a polynomial time $B_{E}$ implies the existence of a polynomial time $B_{O}$.

We suppose it is possible to compute the discrete log of an output of $\mathrm{G}$ after seeing a sequence of all transaction orders. Without loss of generality we can assume success rate is an $\varepsilon$. We will use $\mathrm{B}_{\mathrm{E}}$ by $\mathrm{B}_{\mathrm{O}}$ as a procedure processing in computing feasible by a polynomial time. So, the input parameters for $B_{E}$ is $K_{i}=\left(r_{i}, s_{i}, t_{j}, M_{k}\right)$.

Now $B_{E}$ computes $H_{I}$ and $h\left(r_{I}, m_{I}\right)$ which will be used as input parameters for $B_{O}$ as follows. $\mathrm{B}_{\mathrm{E}}$ chooses $x_{1}, x_{2}, \ldots, x_{k} \in Z_{q}$ at random and computes $y_{1} \equiv g^{x_{1}}, y_{2} \equiv g^{x_{2}}, \ldots, y_{k} \equiv g^{x_{k}}$ Then $\mathrm{B}_{\mathrm{E}}$ has obtained a public message $\mathrm{H}_{\mathrm{I}}=(\mathrm{p}, \mathrm{q}, \mathrm{g}$ 
$\left.\mathrm{y}_{1}, \ldots, \mathrm{y}_{\mathrm{k}-1}, \mathrm{y}_{\mathrm{k}}\right)$. So $\mathrm{B}_{\mathrm{E}}$ computes $y_{i}^{r_{j}} \equiv g^{x_{i} r_{j}}$ for $i=1,2, \ldots, p, \quad j=1,2, \ldots, q$. Thus, uses the $\mathrm{H}_{1}=\left(\mathrm{p}, \mathrm{q}, \mathrm{g}_{1}, \ldots, \mathrm{y}_{\mathrm{k}-1}, \mathrm{y}_{\mathrm{k}}\right)$ and $\mathrm{K}_{\mathrm{i}}=\left(\mathrm{r}_{\mathrm{i}}, \mathrm{s}_{\mathrm{i}}, \mathrm{t}_{\mathrm{j}}, \mathrm{M}_{\mathrm{k}}\right)$ to $\mathrm{B}_{\mathrm{O}}$. In computing feasible condition for any queried transaction order by $B_{0}, B_{E}$ computes by using $\mathrm{H}_{1}=(p, q, g$ $\left.\mathrm{y}_{1}, \ldots, \mathrm{y}_{\mathrm{k}-1}, \mathrm{y}_{\mathrm{k}}\right)$ and sends $\mathrm{h}\left(\mathrm{r}_{\mathrm{r}}, \mathrm{m}_{\mathrm{I}}\right)$ message to $\mathrm{B}_{\mathrm{o}}$. The EC transaction protocol will complete. Finally, $\mathrm{B}_{\mathrm{E}}$ outputs the result of $\mathrm{B}_{\mathrm{O}}$, which is an $1 / \varepsilon$ with non-negligible probability cases. We would find the discrete $\log$ in expected $1 / \varepsilon$ steps. We prove under reasonable assumptions, that our proposed protocol is computationally secure even on public network within different Web-based platforms.

\section{Performance}

In this section, we shall calculate performance of our new method I: The transmission cost on the delivery of the Web-based platform In our method

Step 1: Send $\left(\mathrm{u}_{\mathrm{s}}, \mathrm{R}, \mathrm{e}_{1}, \mathrm{~s}_{1}\right)$ with the request to the transaction supplier $\mathrm{u}_{\mathrm{m}}$.

Step 2:Send $\left(C_{R}, t_{m}, e_{2}, s_{2}\right)$ with the response to the server $u_{s}$. Send the Web-based platform $C_{R}$ and its signature $\left(C_{R}, t_{m}, e_{2}, s_{2}\right)$ to $u_{s}$

II: The computation cost on verifying the signature of an executable program

In our proposed method verifies the signature.

$g^{t_{s} h(M)} \equiv y_{m}\left(r_{2} y_{s}\right)^{r_{2}} \bmod \quad p$

Our method uses 2 modular exponentiation computing time and 1 hash function computing time.

III: The security model comparison in implement.

In our proposed method: The client checks if the signature satisfies the congruence $g^{t_{s} h(M)} \equiv y_{m}\left(r_{2} y_{s}\right)^{r_{2}} \bmod \quad p$

The server checks if the signature satisfies the congruence $\mathrm{e}_{3}=\mathrm{h}\left(\mathrm{r}^{*}, \mathrm{u}_{\mathrm{c}} \| \mathrm{P}\right)$ and $\mathrm{M} \equiv \mathrm{C}_{\mathrm{R}}(\mathrm{P}) \bmod \mathrm{p}$.

\section{Conclusion}

We proposed a protocol that developed to allow clients to get committing Web-based platform signature. In our signature scheme the public key of the Web-based platform owner is used for signature verification that a signature is created from both the public key of the transaction supplier and that of the server Web-based platform owner. In our proposed method, transaction suppliers no use any table to store EC Web-based servers' information that will more efficient and safety than any others EC Web-based systems. Without identify creator, our effective approach on the public keys lead to a verification procedure, and created signatures are checked relatively fast. The most nature extension to this novel protocol scheme is a server-based signature that integrated together with EC application package will allow client and the server to commit with one another. 


\section{References}

1. T. ElGamal, "A public key cryptosystem and a signature ,scheme based on discrete logarithms," IEEE Trans. Inf. Theory, vol.IT-31, no.4, pp.469-472, 1985.

2. C,P, Schnorr, "Efficient identification and signatures for smart Cards," Advances in Cryptology-CRYPTO'89, LNCS 435, pp.239-252, Springer-Veriag, 1990.

3. R.C, Merklc, "A fast software one-way hash function," J. of Cryptology, vol.3, no.1, pp.43-58, 1990.

4. C. Park, "A Fiat-Shamir-like identification protocol without a highly reliable trusted center," Proc. the 1992 Symp. on Cryptography and lnf. Security, no.6D, 1992.

5. Y. Desmedt and Y. Frankel, "Multi-signatures for virus protection," Proc, the 5th lnt'l Computer Virus and Security Conf., 1992.

6. E. Okamoto, "Integrated security system and its application to anti-viral methods," Proc. the 6th Virus and Security Conf, 1993.

7. M. Cyirault, "Self-certified public keys," Advances in Cryptology-EUROCRYP'FO'91, LNCS 547, pp.490-497, Springer-Veriag, 1991.

8. K. Usuda, M. Mambo, T. Uyematsu and E. Okamoto "Proposal of an automatic signature scheme using a Web-based platform”, IEICE Trans. Fundamentals E79-A (1) (1996) 94101

9. K. Nyberg, R.A. Rueppel, "Message recovery for signature scheme based on the discrete logarithm problem," Designs, Codes and Cryptography, No 7, pp.61-81, 1996

10. A. Shamir, "Identity-based cryptosystem based on the discrete logarithm problem," Proc. CRYPTO'84, 1985, pp. 47-53.

11. D. W. Manchala, "E-Commerce Trust Metrics and Models," J. of IEEE Internet Computing, March 2000, pp. 36-44.

12. R. Sherwood, B. Bhattacharjee and A. Srinivasan, "A Protocol for Scalable Anonymous Communication," Proc. the IEEE Symposium on Security and Privacy, 2002, pp.1-12.

13. A. Ginige and S. Murugesan, "Web Engineering: An Introduction," J. of IEEE MultiMedia, January 2001, pp.14-18.

14. J. B. Lim and A. R. Hurson, "Transaction Processing in Mobile, Heterogeneous Database Systems," IEEE Trans. On Knowledge and data Engineering, Vol. 14, No. 6, 2002, pp.1330-1346.

15. Jan, J.K. and Whe Dar Lin :An Efficent Anonymous Channel Protocol in Wireless Communications, IEICE Trans. on Communications, Vol.E84-B, No.3, PP.484-491, 2001.

16. Whe Dar Lin, "Using Marketing Factors Associated with Web site in E-commerce," The International Conference on ASEMA, pp7 11, 2002. 